\title{
Multimodal analgesia with multiple intermittent doses of erector spinae plane block through a catheter after total mastectomy: a retrospective observational study
}

\author{
Boohwi Hong', Seunguk Bang ${ }^{2,3}$, Woosuk Chung ${ }^{1}$, Subin Yoo ${ }^{2,3}$, Jihyun Chung ${ }^{2,3}$, and Seoyeong Kim ${ }^{2,3}$ \\ ${ }^{1}$ Department of Anesthesiology and Pain Medicine, Chungnam National University Hospital, Daejeon, Korea \\ ${ }^{2}$ Department of Anesthesiology and Pain Medicine, Daejeon St. Mary's Hospital, College of Medicine, \\ The Catholic University of Korea, Daejeon, Korea \\ ${ }^{3}$ Department of Anesthesiology and Pain Medicine, College of Medicine, The Catholic University of Korea, Seoul, Korea
}

Background: Although case reports have suggested that the erector spinae plane block (ESPB) may help analgesia for patients after breast surgery, no study to date has assessed its effectiveness. This retrospective observational study analyzed the analgesic effects of the ESPB after total mastectomy.

Methods: Forty-eight patients were divided into an ESPB group $(\mathrm{n}=20)$ and a control group $(\mathrm{n}=28)$. Twenty patients in the control group were selected by their propensity score matching the twenty patients in the ESPB group. Patients in the ESPB group were injected with $30 \mathrm{~mL}$ 0.375\% ropivacaine, followed by catheter insertion for further injections of local anesthetics every 12 hours. Primarily, total fentanyl consumption was compared between the two groups during the first 24 hours postoperatively. Secondary outcomes included pain intensity levels (visual analogue scale) and incidence of postoperative nausea and vomiting (PONV).

Results: Median cumulative fentanyl consumption during the first 24 hours was significantly lower in the ESPB (33.0 $\mu$; interquartile range [IQR], 27.0-69.5 $\mu \mathrm{g})$ than in the control group $(92.8 \mu \mathrm{g}$; IQR, 40.0-155.0 $\mu \mathrm{g})(P=$ $0.004)$. Pain level in the early postoperative stage $(<3 \mathrm{hr})$ and incidence of PONV ( $0 \%$ vs. $55 \%)$ were also significantly lower in the ESPB group compared to the control $(P=0.001)$.

Conclusions: Intermittent ESPB after total mastectomy reduces fentanyl consumption and early postoperative pain. ESPB is a good option for multimodal analgesia after breast surgery. (Korean J Pain 2019; 32: 206-14)

Key Words: Acute Pain; Analgesia; Anesthesia, Conduction; Breast; Mastectomy; Nerve Block; Pain, Postoperative; Ropivacaine; Ultrasonography.

Received January 14, 2019. Revised April 9, 2019. Accepted April 15, 2019.

Correspondence to: Seunguk Bang

Department of Anesthesiology and Pain Medicine, Daejeon St. Mary's Hospital, College of Medicine, The Catholic University of Korea, 64 Daeheung-ro, Jung-gu, Daejeon 34943, Korea

Tel: +82-42-220-9046, Fax: +82-42-252-6807, E-mail: seungukb@naver.com

ORCID: https://orcid.org/0000-0001-6609-7691

Previous presentation at conferences: The 67th Korean Pain Society of Scientific Meeting, 18th Nov. 2018, Seoul, Korea.

(a) This is an open-access article distributed under the terms of the Creative Commons Attribution Non-Commercial License (http:// creativecommons.org/licenses/by-nc/4.0/), which permits unrestricted non-commercial use, distribution, and reproduction in any medium, provided the original work is properly cited.

(C) The Korean Pain Society, 2019 


\section{INTRODUCTION}

Although breast innervation has been reported to vary, the medial and lateral aspects of the breast are most frequently innervated by the anterior and lateral cutaneous branches of the T2-T6 intercostal nerves, with infrequent involvement of the $\mathrm{Tl}$ and $\mathrm{T} 7$ intercostal nerves [1]. The superior aspect of the breast is most frequently innervated by the supraclavicular branch of the superficial cervical plexus [1]. Moreover, although the lateral and medial pectoral nerves do not innervate the subcutaneous tissue of the breast, these nerves contribute to pain during and after breast surgery through disruption, stretching, or spasms of the pectoral muscles or fascia [1].

Thus, multimodal analgesia after breast surgery usually consists of the thoracic epidural block (TEB) or thoracic paravertebral nerve block (TPVB), both of which act primarily by blocking nerves aforementioned. Although these nerve blocks have been shown effective, they are relatively difficult to perform and have several drawbacks. For example, the adverse effects of the TEB include hypotension, bradycardia, motor blockade, hematoma, and abscess [2-5]. Although the TPVB can overcome these drawbacks of the $\mathrm{TEB}$, these drawbacks cannot be completely eliminated [6]. In addition, the TPVB not only requires multiple injection but also is difficult to maintain the position of the catheter, and the risk of pneumothorax exists [7-9].

The erector spinae plane block (ESPB) is a technically simple procedure, with easily identified sonographic landmarks, for analgesia in patients undergoing thoracic, abdominal, lumbar, and urologic surgery [10-18]. The ESPB can provide extensive ranges of ventral and dorsal ramus blockade from T1-2 to T8-12 and allows easy insertion of a catheter into the distension induced by the injection $[12,13,19,20]$. Moreover, the ESPB is performed away from the pleura and neuraxis, reducing the risks of complications associated with these structures.

The sensory blockade capabilities of the ESPB and the nervous innervation of the breast suggest that the ESPB can be considered as an option for multimodal analgesia after total mastectomy. Therefore, this study assessed the analgesic effects of the ESPB as part of the multimodal analgesic procedure after total mastectomy. We hypothesized that the ESPB would reduce opioid consumption during the first 24 hours after total mastectomy.

\section{MATERIALS AND METHODS}

This retrospective cohort study was approved by the Institutional Review Board of Institutional Review Board of The Catholic University of Korea, Daejeon St. Mary's Hospital (DCl8ZESIO054). The electronic medical records of 77 patients (American Society of Anesthesiologists Physical Status 1 and 2) who received total mastectomy at Daejeon St. Mary's Hospital between January 1, 2017, and June 30, 2018, were retrospectively reviewed. Patients were excluded if they had a body mass index (BMI) >35 $\mathrm{kg} / \mathrm{m}^{2}$, were diabetic or had other neuropathies, or had neurologic or psychiatric problems. Patients were also excluded if they were taking opioids as a chronic analgesic treatment prior to surgery, if they developed postoperative cognitive dysfunction or delirium, or if they refused $\mathrm{pa}-$ tient-controlled analgesia (PCA). Also excluded were patients who underwent bilateral breast surgery and extended mastectomy, including pectoralis major or minor muscle excision, or mastectomy with simultaneous reconstructive surgery using a muscle flap or implant. Patients were divided into two groups: a control group consisting of patients who received the conventional acute pain service (APS) protocol of our center, and an ESPB group which consisted of patients who had the ESPB along with the conventional APS protocol.

\section{Perioperative anesthesia care protocol}

Total intravenous anesthesia was induced with a target effect-site concentration of propofol $3.0-3.5 \mu \mathrm{g} / \mathrm{mL}$ and remifentanil 4-6 ng/mL and continuous intravenous infusion of rocuronium, adjusted to maintain a bispectral index of 40-60. All patients were administered intravenous ketorolac $30 \mathrm{mg}$ and fentanyl $1 \mu \mathrm{g} / \mathrm{kg}$ at the end of surgery. Upon emergence, the patients were transferred to the post-anesthesia care unit (PACU), where the pain was assessed and controlled based on the APS protocol of The Catholic University of Korea, Daejeon St. Mary's Hospital for breast surgery.

The APS protocol for postoperative multimodal analgesia consists of oral medication, intravenous fentanyl with PCA, and rescue analgesics. Patients received oral acetaminophen $650 \mathrm{mg}$ three times a day for 5 days after surgery. Also, intravenous fentanyl with PCA was started in the PACU. The PCA pump (Hospira GemStar ${ }^{\circledR}$ Pump; 
Hospira Inc., Lake Forest, IL) was programmed to administer a bolus dose of $0.5 \mu \mathrm{g} / \mathrm{kg}$, without background infusion, with a lockout time of 7 minutes. Patients with persistent postoperative pain greater than a score of 4 on the visual analogue scale (VAS) were prescribed intravenous tramadol $25 \mathrm{mg}$ as a rescue analgesic.

Prior to surgery, all patients undergoing total mastectomy were provided sufficient instructions on how to report pain levels by VAS ( $0 \mathrm{~cm}$, no pain; $10 \mathrm{~cm}$, worst pain) and to press the bolus button when VAS score was $\geq 4$. The patients were reinstructed in the ward by attending nurses.

\section{ESPB procedure}

All the ESPBs were performed by a skillful expert in ultrasound (US)-guided nerve blocks (author S.B.) upon the induction of general anesthesia to minimize the pain and discomfort during the procedure [21]. After general anesthesia, the patient was placed in the lateral position. A US-guided ESPB was performed using a sterile technique. Using a 5-12 MHz linear probe and manually counting from the first to the twelfth rib, the level of the $\mathrm{T} 5 \mathrm{rib}$ and transverse process (TP) was identified and marked on the skin. At this level, the probe was moved transversely from a lateral to medial position in order to detect any changes in shape that traversed the rib or TP. At the point where the round shadow of the rib shifted to the rectangular shape of the TP, an 18-gauge Tuohy needle was inserted with an in-plane approach.

After the needle penetrated three muscles (the trapezius, rhomboid major, and erector spinae), it was located in the fascial plane between the TP and erector spinae muscle (Fig. 1). The fascial plane was confirmed to be well-separated by injecting $2 \mathrm{~mL}$ of saline, followed by the

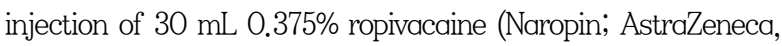
Luton, UK) containing epinephrine $(1: 200,000)$ and the insertion of a catheter 2 to $3 \mathrm{~cm}$ over the tip of the Tuohy needle under real-time US guidance. In the ward, patients undergoing the ESPB were manually injected with $30 \mathrm{~mL}$ $0.375 \%$ ropivacaine containing epinephrine $1: 200,000$, through the indwelling ESP catheter every 12 hours. The ESP catheter was usually kept for 2-3 days and then
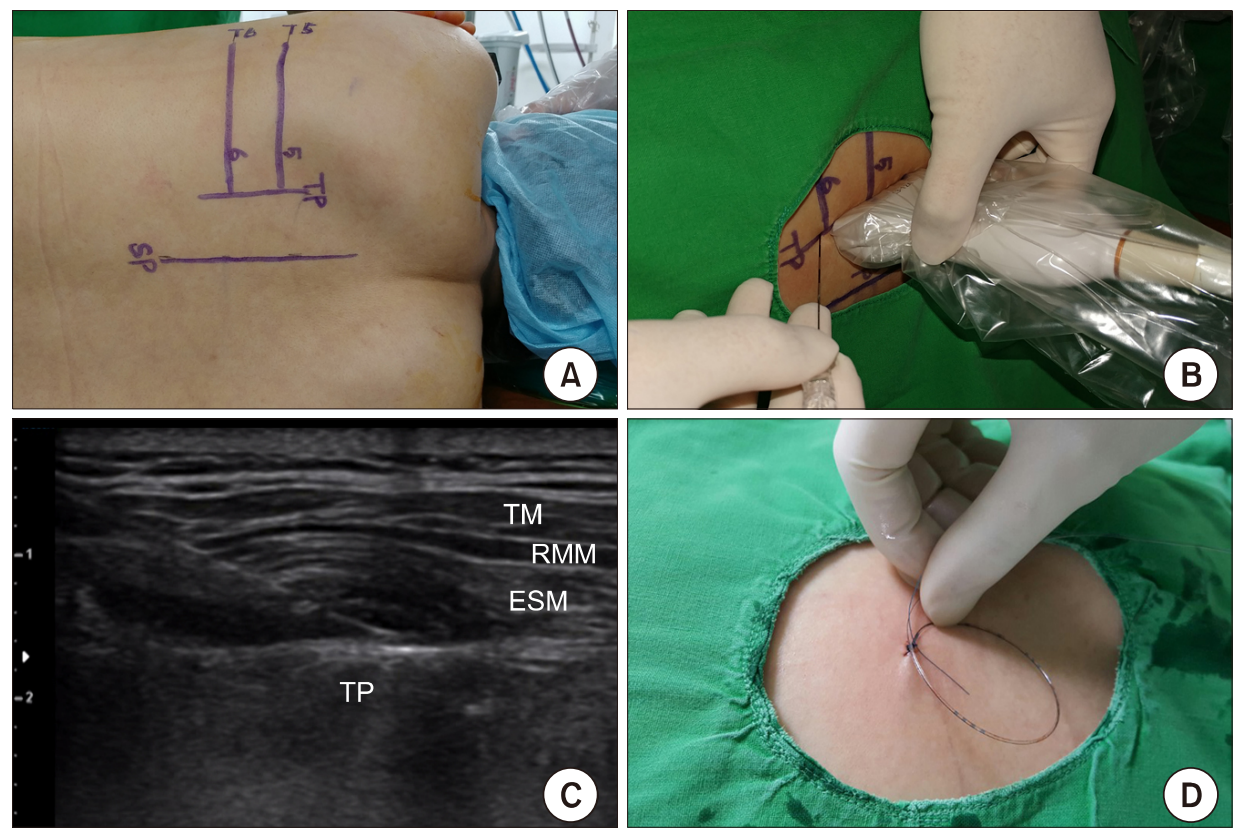

Fig. 1. The erector spinae plane block. (A) The level of the T5 rib and transverse process was located using a counting-down approach from the first rib; this was marked on the skin at the lateral position. (B) After placing a linear probe parallel to the vertebral axis, a needle was inserted toward the transverse process. (C) After confirming proper position of needle tip, we injected the local anesthetic. The catheter was inserted using real-time ultrasound guidance. (D) The catheter was secured by suture to the skin. ESM: erector spinae muscle, RMM: rhomboid major muscle, TM: trapezius muscle, TP: transverse process, SP: spinous process of vertebra. Data from the article of Kwon et al. (J Korean Med Sci. 2018; 33: e291) [32]. 
removed.

\section{Outcome measures}

The primary outcome was the total amount of fentanyl consumed for 24 hours, as determined by a PCA program that transferred the time and number of fentanyl boluses to the APS electronic recoding sheet. The secondary outcome was the 24 hours postoperative VAS pain score, as recorded by ward nurses on an electronic pain chart. Other factors recorded included patient demographic characteristics, such as age, weight, height, and BMI. Perioperative characteristics included the volume of intraoperative fluids administered, the duration of surgery and anesthesia, and if axial lymph node dissection (ALND) was performed.

Complications during the ESPB (e.g., vascular and pleural punctures) and any symptoms which appeared during the injection through the indwelling catheter were noted. Opioid-related side effects, including postoperative nausea, pruritus, and respiratory depression were also assessed.

\section{Statistical analysis}

All statistical analyses were performed using $\mathrm{R}$ software (vers. 3.4.4; R Project for Statistical Computing, Vienna, Austria). Propensity score matching was performed using the Matchlt package in $\mathrm{R}$ to account for the differences in baseline characteristics [22]. The logistic regression model was used to calculate the propensity scores. The dependent variable is set as a binary response with the ESPB group as 1 and the control group as 0 , and the logistic regression analysis was performed by designating the covariate (ALND, age, height, and weight) to be corrected as an independent variable. Especially, the ALND was included in the covariate because it is a factor that directly affects postoperative pain.

The logistic regression analysis estimates the propensity score model, and the probability that each subject will be included in the control group by the given covariance corresponds to the propensity score. We performed nearest neighbor matching. This method is matching the absolute differences of the estimated propensity score differences of all subjects in both groups starting with the smallest. The normality of continuous data was assessed using the Shapiro-Wilk test. If normality was satisfied, comparisons between groups were determined by independent $t$-tests, with the results expressed as mean \pm standard deviation. If normality was not satisfied, groups were compared using the Mann-Whitney $U$-test and the results were expressed as a median value (interquartile range, IQR) [23].

Point estimation and the confidence interval (CI) of the Hodges-Lehmann's median were calculated using SAS software (ver. 9.3 for Windows; SAS Korea, Seoul, Korea). Categorical data were analyzed using the chi-squared test or Fisher's exact test, as appropriate. For all calculations, a two-tailed $P$ value $<0.05$ was considered statistically significant. Repeated measures analysis of variance was used to analyze the differences between the groups over time. If differences were significant, Bonferroni's correction was applied to minimize the probability of type I error that occurs when multiple tests are performed for each point.

\section{RESULTS}

During the study period 77 patients underwent total mastectomy; of these, 29 were excluded, including 21 with incomplete records, four male patients, one patient with dementia, one patient who underwent immediate reconstructive surgery with an abdominal muscle flap, one who underwent contralateral side surgery, and one who refused PCA. Of the remaining 48 patients, 20 underwent the ESPB and 28 did not. Of these 28 control patients, 20 were selected via propensity score matching (Fig. 2). The demographic and clinical characteristics of the two groups are shown in Table 1. The anesthesia time was significantly longer in the ESPB than in the control group $(P=0.014)$.

The two groups differed in opioid consumption during the first postoperative 24 hours, with a significant intergroup interaction $(P=0.005)$ (Fig. 3). Median cumulative fentanyl consumption over 24 hours was significantly lower in the ESP group $(33.0 \mu \mathrm{g}$; IQR, 27.0-69.5 $\mu \mathrm{g})$ than in the control group (92.8 $\mu \mathrm{g}$; IQR, 40.0-155.0 $\mu \mathrm{g})$ group $(P=$ 0.004), with a Hodges-Lehman median difference of $51 \mu \mathrm{g}$ (95\% CI, 8-94 $\mu \mathrm{g}$ ) between the two groups. The VAS pain score also changed over time, with a significant intergroup interaction $(P<0.001)$ (Fig. 4). The VAS score was lower in the ESP group during the early postoperative period. At 3 hours, the score differed significantly in the two groups, but the difference became insignificant after propensity score 


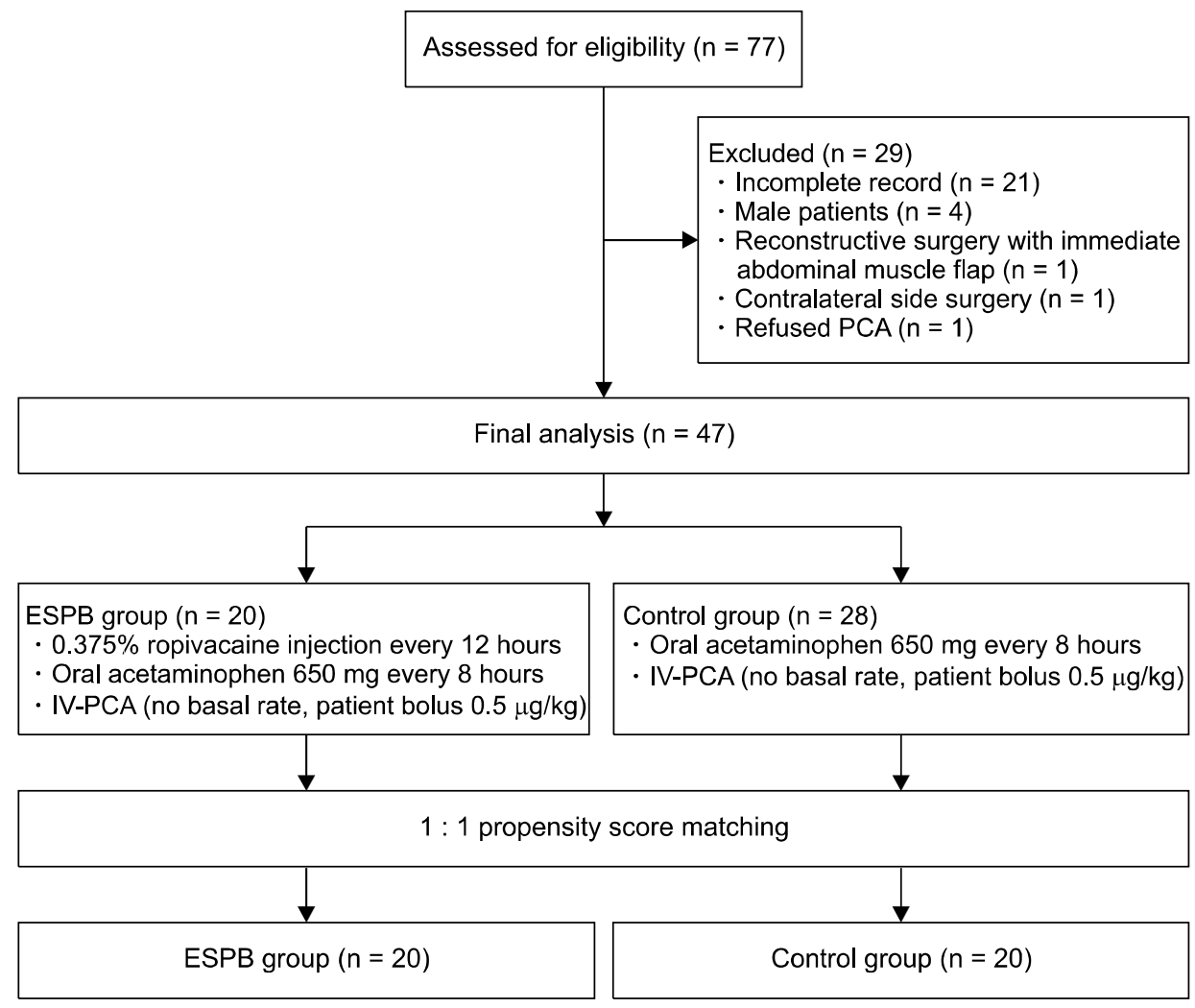

Fig. 2. Flow chart of patient selection and propensity score matching. PCA: patient-controlled analgesia, ESPB: erector spinae plane block, IV: intravenous.

Table 1. Demographic and Clinical Characteristics of Study Patients before and after Propensity Score Matching

\begin{tabular}{|c|c|c|c|c|}
\hline \multirow{2}{*}{ Variable } & \multicolumn{2}{|c|}{ Before matching } & \multicolumn{2}{|c|}{ After matching } \\
\hline & $\operatorname{ESPB}(n=20)$ & Control $(n=28)$ & $\operatorname{ESPB}(n=20)$ & Control $(n=20)$ \\
\hline Age (yr) & $57.9 \pm 12.7$ & $57.2 \pm 11.6$ & $57.9 \pm 12.7$ & $57.0 \pm 11.7$ \\
\hline Height $(\mathrm{cm})$ & $156.2 \pm 5.4$ & $157.1 \pm 5.1$ & $156.2 \pm 5.4$ & $157.6 \pm 5.1$ \\
\hline Weight (kg) & $57.7 \pm 8.0$ & $57.0 \pm 7.8$ & $57.7 \pm 8.0$ & $57.3 \pm 8.6$ \\
\hline BMI $\left(\mathrm{kg} / \mathrm{m}^{2}\right)$ & $22.7(21.0-24.3)$ & $22.7(21.0-24.3)$ & $22.7(21.0-24.3)$ & $22.6(21.0-24.9)$ \\
\hline Intraoperative fluid $(\mathrm{mL})$ & $430.0(210.0-685.0)$ & $500.0(425.0-700.0)$ & $430.0(210.0-685.0)$ & $550.0(450.0-750.0)$ \\
\hline Surgery time (min) & $126.0(108.5-154.5)$ & $110.5(96.0-132.5)$ & $126.0(108.5-154.5)$ & $110.5(94.0-141.5)$ \\
\hline Anesthesia time (min) & $169.0(146.0-189.5)$ & $137.5(115.5-161.0)$ & $169.0(146.0-189.5)$ & $139.5(115.0-164.0)$ \\
\hline ALND & $4(20.0)$ & $9(32.1)$ & $4(20.0)$ & $4(20.0)$ \\
\hline
\end{tabular}

Values are presented as mean \pm standard deviation, median (interquartile range), or number (\%).

ESPB: erector spinae plane block, BMI: body mass index, ALND: axillary lymph node dissection.

matching. Additionally, the incidence of PONV was significantly higher in the control group $(P<0.001$, Table 2).

Sonographic visualization throughout the ESPB procedure showed no evidence of vascular or pleural puncture or of epidural or intrathecal injection in any patient, and none reported systemic toxicity or intrathecal injection signs. Complaints reported postoperatively during injection of local anesthetic solution through the indwelling catheter included transient, mild injection pain ( $n=7)$, cooling sensation $(n=2)$, and flowing sensation in the back $(n=5)$. Symptoms of local anesthetics systemic toxicity (LAST), catheter related problem (accidental catheter removal, infection) and clinically problematic hemodynamic changes were not reported. 


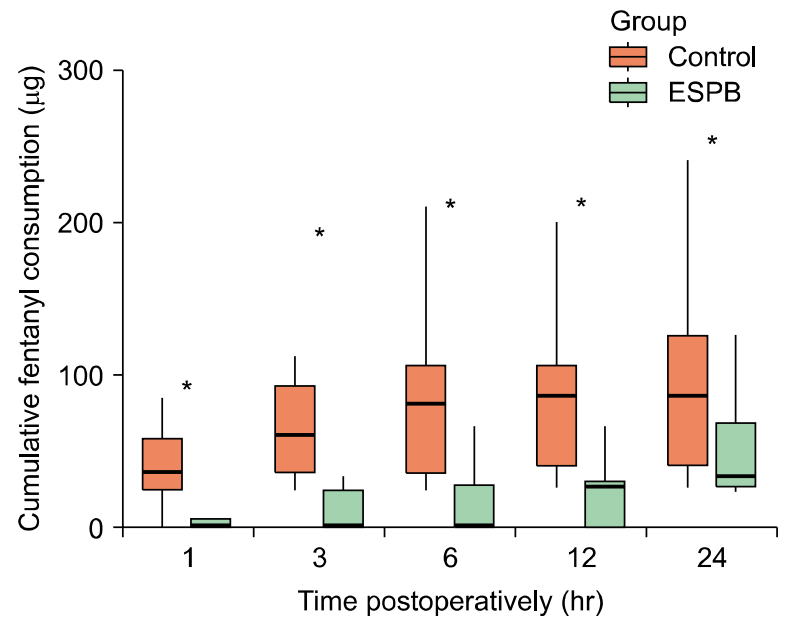

Fig. 3. Cumulative fentanyl consumption over time in the erector spinae plane block (ESPB) and control groups. Cumulative fentanyl consumption at all postoperative time points was lower in the ESPB than in the control group. Data are expressed as median (interquartile range). ${ }^{\star} P<$ 0.01 .

Table 2. Postoperative Analgesia Outcomes and Incidence of PONV

\begin{tabular}{|c|c|c|c|}
\hline Variable & $\begin{array}{c}\text { ESPB } \\
(n=20)\end{array}$ & $\begin{array}{l}\text { Control } \\
(n=20)\end{array}$ & $P$ value \\
\hline Frequency of rescue analgesics & & & 0.072 \\
\hline 1 & $1(5.0)$ & $0(0)$ & \\
\hline 2 & $0(0)$ & $4(20.0)$ & \\
\hline PONV & $0(0)$ & $11(55.0)$ & $<0.001$ \\
\hline
\end{tabular}

Values are presented as number (\%). Patients with persistent postoperative pain greater than a score of 4 on a visual analogue scale were prescribed intravenous tramadol $25 \mathrm{mg}$ as a rescue analgesic.

ESPB: erector spinae plane block, PONV: postoperative nausea and vomiting.

\section{DISCUSSION}

The objective of our study was to assess the effect of the ESPB as multimodal analgesia after total mastectomy with sentinel lymph node dissection (SLND). The study found that total fentanyl consumption at postoperative hours 1 , $3,6,12$, and 24 was lower in the ESPB group than in the control group. The median cumulative amount of fentanyl consumed for 24 hours after surgery was $33 \mu \mathrm{g}$ in the ESPB group and $92.8 \mu \mathrm{g}$ in the control group, with morphine equivalent doses of $3.3 \mathrm{mg}$ and $10 \mathrm{mg}$, respectively. These results suggest that ESPB reduces opioid consumption by about $65 \%$, providing effective analgesia in

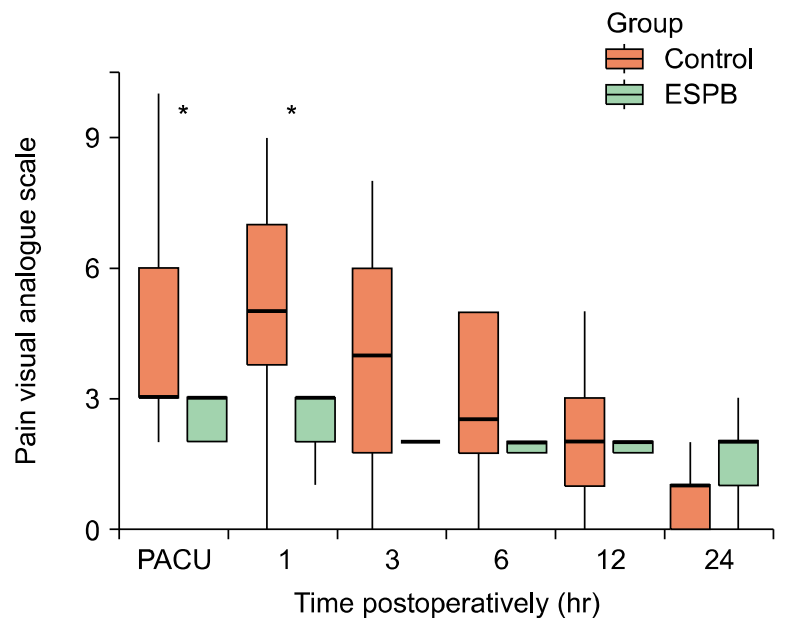

Fig. 4. Pain visual analogue scales over time in the erector spinae plane block (ESPB) and control groups. Pain intensity at an early postoperative stage was significantly lower in the ESPB than in the control group. Data are expressed as median (interquartile range). PACU: post-anesthesia care unit. ${ }^{\star} P<0.008$.

mastectomy patients.

Previous case reports have also described that the ESPB is effective in analgesia after breast surgery. For example, the ESPB provided effective analgesia with minimal morphine consumption in patients who underwent total mastectomy and ALND with a tissue expander [24], and in patients who underwent various types of mastectomy with SLND or ALND [25]. In a recent randomized controlled trial, a single $20 \mathrm{~mL}$ ESPB injection before general anesthesia in breast surgery patients, including patients undergoing modified mastectomy, simple (total) mastectomy, and lumpectomy, was found to reduce cumulative opioid consumption after 6,12 , and 24 hours and total morphine consumption at 24 hours, the latter by $65 \%$ [26].

The present study differs from previous studies in several ways. First, unlike the study performed in the condition of various breast surgery [26], our study was restricted to one kind of total mastectomy. In addition, propensity score matching was applied to patients receiving ALND, which has a great effect on postoperative pain, to maintain homogeneity between groups. Second, our study assessed the efficacy of intermittent analgesia with ESPB administered using a catheter. Although single shot injections as a part of multimodal framework is effective analgesia in the early postoperative period after the surgery, it is not yet known if they provide adequate late analgesia. 
In contrast, continuous infusion or intermittent bolus has been tried as an option for effective analgesia in the late postoperative period [27,28], and maintaining the catheter for 2-3 days is advantageous in controlling the breakthrough pain which occurs during the late postoperative period due to ambulation, coughing, dressing change, and more. Breakthrough pain can proceed to chronic pain by direct peripheral sensitization and central sensitization in exposure to repeated pain. In such cases, continuous and aggressive control of breakthrough pain using a catheter may be beneficial. Third, to our knowledge, this study is the first to show that the ESPB reduces the incidence of PONV, suggesting the opioid-sparing effect of the ESPB contributed to the lower incidence of PONV in the ESPB group [29,30].

ESPB may provide effective analgesia in the breast by wide sensory blockade of the anterior and lateral cutaneous branches of the spinal nerves that innervate breast tissue, a finding observed in several case reports. In one report, two patients who underwent an ESPB with 0.375\% ropivacaine $20 \mathrm{~mL}$ at the $\mathrm{T} 5$ level, after total mastectomy and breast reconstruction surgery, showed sensory blockade at the T2-T8 and T3-T7 dermatome levels [31]. Other studies have reported that an ESPB with a $20-30 \mathrm{~mL}$ bolus at the T4-5 level resulted in sensory blockade at either T2-T8 or T2-T10, as confirmed by pin prick or ice cold tests $[13,14,32,33]$.

Although the mechanism of the ESPB remains unclear, local anesthetics injected beneath the erector spinae muscle (interfacial injection) can spread through the costotransverse foramen and peripheral porous tissues into the intercostal and thoracic paravertebral spaces (TPVS) [12,34]. The costotransverse foramen is a bony gap between the neck of a rib and the TP of a vertebra. A cadaveric study reported that tracer dyes injected beneath the erector spinae muscle can spread into the deep structures of the external intercostal muscle and internal intercostal membrane through the costotransverse foramen [12].

Conventionally, the superior costotransverse ligament was assumed to be a compact ligament and that an effective TPVB could only be achieved after direct penetration of the ligament. However, the TPVS and intercostal nerves could be stained even if the dye was injected above the superior costotransverse ligament or intercostal muscle through the porous tissue around the superior costotransverse ligament [35]. Another study reported gaps between the medial and lateral portions of the superior costotransverse ligament [36]. Similar dye spreading patterns to the TPVS were also reported in retrolaminar blocks, another alternative to paravertebral blocks, without pleural displacement or superior costotransverse ligament penetration $[34,37]$.

In contrast, recent cadaveric studies have reported that local anesthetics tend to spread to the intercostal rather than the TPVS to block multiple intercostal nerves. Thus, it remains unclear whether the primary analgesic mechanism of the ESPB depends on the spreading of local anesthetics to the TPVS. Because the breast is a subcutaneous organ, the analgesic coverage of the ESPB does not need to extend to the sympathetic chain ganglions. Nerve blockade of the ventral rami is sufficient to provide efficient analgesia by blocking somatic pain.

This study had several limitations. First, its retrospective design and the absence of randomization and blinding may have resulted in potential selection and information biases. Also, we could not rule out the expectation that those who have a catheter will have better pain control. Second, the manual injection of local anesthetics may have affected the spreading of the local anesthetics due to different infusion rates and pressure. In addition, it is not clear that the amount of local anesthetic was maintained below the toxic threshold.

Because the ESPB has only recently been introduced, no study has been previously published examining plasma concentrations. However, some studies have been discussed the maximal plasma concentration (Cmax) in the transversus abdominis plane block, another interfascial plane block as the ESPB, to be $1.56 \mu \mathrm{g} / \mathrm{mL}$ when $150 \mathrm{mg}$ of $0.75 \%$ ropivacaine was administered to the plane. This level of plasma concentration is lower than $2.2 \mu \mathrm{g} / \mathrm{mL}$ which is known as the plasma concentration that can cause systemic toxicity [38,39]. In the present study, we used $30 \mathrm{~mL}$ of $0.375 \%$ ropivacaine $(112.5 \mathrm{mg}$ ) because it is reported that large local anesthetic volumes $(30 \mathrm{~mL}$ ) would be essential to achieve regional anesthesia and analgesia in a retrolaminar block to similar to the ESPB [37]. It does not exceed the maximum recommended doses of local anesthetics, and we expected that the addition of $\mathrm{ep}^{-}$ inephrine to the local anesthetic may provide the margin of safety because epinephrine decreases the systemic absorption and the maximum plasma concentrations even for ropivacaine [38]. Consequently, none of the patients 
showed signs of LAST. Furthermore, there have been several studies which involved the use of $20-30 \mathrm{~mL}$ of ropivacaine for the ESPB [39]. Finally, because PCA data were obtained only for 24 hours, the long-term analgesic effects of the ESPB cannot be evaluated in our study.

In conclusion, the present study supports the use of the intermittent ESPB after total mastectomy with SLND as a safe analgesic procedure that may reduce opioid consumption and PONV. Further prospective studies with controlled variables and specific standard pain management protocols are warrented to compare the ESPB to other conventional block techniques, such as the TPVB and pectoralis block, performed to alleviate postoperative pain after breast surgery.

\section{ACKNOWLEDGMENTS}

This research was supported by Chungnam National University Hospital Research Fund, 2018.

\section{CONFLICT OF INTEREST}

No potential conflict of interest relevant to this article was reported.

\section{ORCID}

Boohwi Hong, https://orcid.org/0000-0003-2468-9271

Seunguk Bang, https://orcid.org/0000-0001-6609-7691

Woosuk Chung, https://orcid.org/0000-0002-6409-2325

Subin Yoo, https://orcid.org/0000-0001-6487-2914

Jihyun Chung, https://orcid.org/0000-0003-1672-7442

Seoyeong Kim, https://orcid.org/0000-0002-2313-3258

\section{REFERENCES}

1. Woodworth GE, Ivie RMJ, Nelson SM, Walker CM, Maniker RB. Perioperative breast analgesia: a qualitative review of anatomy and regional techniques. Reg Anesth Pain Med 2017; 42: 609-31.

2. Wildsmith JA. Continuous thoracic epidural block for surgery: gold standard or debased currency? Br J Anaesth 2012; 109: 9-12.

3. Choe WJ, Kim JY, Yeo HJ, Kim JH, Lee SI, Kim KT, et al. Postpartum spinal subdural hematoma: irrelevant epidural blood patch: a case report. Korean J Anesthesiol 2016; 69: 189-92.
4. Yeung $\mathrm{JH}$, Gates S, Naidu BV, Wilson MJ, Gao Smith F. Paravertebral block versus thoracic epidural for patients undergoing thoracotomy. Cochrane Database Syst Rev 2016; 2: CD009121.

5. Rawal N. Epidural technique for postoperative pain: gold standard no more? Reg Anesth Pain Med 2012; 37 : 310-7.

6. Marhofer D, Marhofer P, Kettner SC, Fleischmann E, Prayer $D$, Schernthaner $M$, et al. Magnetic resonance imaging analysis of the spread of local anesthetic solution after ultrasound-guided lateral thoracic paravertebral blockade: a volunteer study. Anesthesiology 2013; 118: 1106-12.

7. Sherwin A, Buggy DJ. Anaesthesia for breast surgery. BJA Educ 2018; 18: 342-8.

8. Pace MM, Sharma B, Anderson-Dam J, Fleischmann K, Warren L, Stefanovich P. Ultrasound-guided thoracic paravertebral blockade: a retrospective study of the incidence of complications. Anesth Analg 2016; 122: 1186-91.

9. Lönnqvist PA, MacKenzie J, Soni AK, Conacher ID. Paravertebral blockade. Failure rate and complications. Anaesthesia 1995; 50: 813-5.

10. Bonvicini D, Giacomazzi A, Pizzirani E. Use of the ultrasound-guided erector spinae plane block in breast surgery. Minerva Anestesiol 2017; 83: 1111-2.

11. Finneran JJ 4th, Gabriel RA, Khatibi B. Erector spinae plane blocks provide analgesia for breast and axillary surgery: a series of 3 cases. Reg Anesth Pain Med 2018; 43: 101-2.

12. Forero M, Adhikary SD, Lopez H, Tsui C, Chin KJ. The erector spinae plane block: a novel analgesic technique in thoracic neuropathic pain. Reg Anesth Pain Med 2016; 41: 621-7.

13. Scimia P, Basso Ricci E, Droghetti A, Fusco P. The ultrasound-guided continuous erector spinae plane block for postoperative analgesia in video-assisted thoracoscopic lobectomy. Reg Anesth Pain Med 2017; 42: 537.

14. Forero M, Rajarathinam M, Adhikary S, Chin KJ. Erector spinae plane (ESP) block in the management of post thoracotomy pain syndrome: a case series. Scand J Pain 2017; 17: 325-9.

15. Chin KJ, Adhikary S, Sarwani N, Forero M. The analgesic efficacy of pre-operative bilateral erector spinae plane (ESP) blocks in patients having ventral hernia repair. Anaesthesia 2017; 72: 452-60.

16. Kline J, Chin KJ. Modified dual-injection lumbar erector spine plane (ESP) block for opioid-free anesthesia in multilevel lumbar laminectomy. Korean J Anesthesiol 2019; 72: 18890.

17. Kim E, Kwon W, Oh S, Bang S. The erector spinae plane block for postoperative analgesia after percutaneous nephrolithotomy. Chin Med J (Engl) 2018; 131: 1877-8.

18. Bang S. Erector spinae plane block: an innovation or a delusion? Korean J Anesthesiol 2019; 72: 1-3.

19. Ramos J, Peng P. Forero M. Long-term continuous erector 
spinae plane block for palliative pain control in a patient with pleural mesothelioma. Can J Anaesth 2018; 65: 852-3.

20. Chin KJ, Malhas L, Perlas A. The erector spinae plane block provides visceral abdominal analgesia in bariatric surgery: a report of 3 cases. Reg Anesth Pain Med 2017; 42: 372-6.

21. Masaracchia MM, Herrick MD, Seiffert EA, Sites BD. Nerve blocks under general anesthesia: time to liberalize indications? Reg Anesth Pain Med 2017; 42: 299-301.

22. Ho D, Imai K, King G, Stuart EA. Matchlt: nonparametric preprocessing for parametric causal inference. J Stat Softw 2011; 42: 1-28.

23. In J, Lee S. Statistical data presentation. Korean J Anesthesiol 2017; 70: 267-76.

24. Kumar A, Hulsey A, Martinez-Wilson H, Kim J, Gadsden J. The use of liposomal bupivacaine in erector spinae plane block to minimize opioid consumption for breast surgery: a case report. A A Pract 2018; 10: 239-41.

25. Tanaka N, Ueshima H, Otake H. Erector spinae plane block for combined lovectomy and radical mastectomys. J Clin Anesth 2018; 45: 27-8.

26. Gürkan Y, Aksu C, Kuş A, Yörükoğlu UH, Kiliç CT. Ultrasound guided erector spinae plane block reduces postoperative opioid consumption following breast surgery: a randomized controlled study. J Clin Anesth 2018; 50: 65-8.

27. Yoshizaki M, Murata H, Ogami-Takamura K, Hara T. Bilateral erector spinae plane block using a programmed intermittent bolus technique for pain management after Nuss procedure. J Clin Anesth 2019. doi: 10.1016/j.jclinane.2019.03.014.

28. Tiburzi C, Cerotto V, Gargaglia E, Carli L, Gori F. Pectoral nerve block $\|$ with programmed intermittent bolus of local anesthetic and postoperative pain relief in breast surgery. Minerva Anestesiol 2019; 85: 201-2.

29. Öbrink E, Jildenstål P, Oddby E, Jakobsson JG. Postoperative nausea and vomiting: update on predicting the probability and ways to minimize its occurrence, with focus on ambulatory surgery. Int J Surg 2015; 15: 100-6.
30. Porreca F, Ossipov MH. Nausea and vomiting side effects with opioid analgesics during treatment of chronic pain: mechanisms, implications, and management options. Pain Med 2009; 10: 654-62.

31. Ohgoshi Y, Ikeda T, Kurahashi K. Continuous erector spinae plane block provides effective perioperative analgesia for breast reconstruction using tissue expanders: a report of two cases. J Clin Anesth 2018; 44: 1-2.

32. Kwon WJ, Bang SU, Sun WY. Erector spinae plane block for effective analgesia after total mastectomy with sentinel or axillary lymph node dissection: a report of three cases. J Korean Med Sci 2018; 33: e291.

33. Kim D, Bang S, Sun WY. Erector spinae plane block with sedation for surgical anesthesia in breast conserving surgery. J Clin Anesth 2019. doi: 10.1016/j.jclinane.2019.03. 003.

34. Adhikary SD, Bernard S, Lopez H, Chin KJ. Erector spinae plane block versus retrolaminar block: a magnetic resonance imaging and anatomical study. Reg Anesth Pain Med 2018; 43: 756-62.

35. Costache I, de Neumann L, Ramnanan CJ, Goodwin SL, Pawa A, Abdallah FW, et al. The mid-point transverse process to pleura (MTP) block: a new end-point for thoracic paravertebral block. Anaesthesia 2017; 72: 1230-6.

36. Luyet C, Eichenberger U, Greif R, Vogt A, Szücs Farkas Z, Moriggl B. Ultrasound-guided paravertebral puncture and placement of catheters in human cadavers: an imaging study. Br J Anaesth 2009; 102: 534-9.

37. Damjanovska M, Stopar Pintaric T, Cvetko E, Vlassakov K. The ultrasound-guided retrolaminar block: volume-dependent injectate distribution. J Pain Res 2018; 11: 293-9.

38. El-Boghdadly K, Pawa A, Chin KJ. Local anesthetic systemic toxicity: current perspectives. Local Reg Anesth 2018; 11 : 35-44.

39. De Cassai A, Bonvicini D, Correale C, Sandei L, Tulgar S, Tonetti T. Erector spinae plane block: a systematic qualitative review. Minerva Anestesiol 2019; 85: 308-19. 